\title{
Design of photonic crystal optical microcavities
}

Jelena Vuckovic, Marko Loncar, Axel Scherer

Jelena Vuckovic, Marko Loncar, Axel Scherer, "Design of photonic crystal optical microcavities," Proc. SPIE 4283, Physics and Simulation of Optoelectronic Devices IX, (9 July 2001); doi: 10.1117/12.432592

SPIE. Event: Symposium on Integrated Optics, 2001, San Jose, CA, United States 


\title{
Design of Photonic Crystal Optical Microcavities
}

\author{
Jelena Vučković, Marko Lončar and Axel Scherer \\ Department of Electrical Engineering \\ California Institute of Technology, 136-93 \\ Pasadena, CA 91125, U.S.A.
}

\begin{abstract}
Using the 3-D finite-difference time-domain (FDTD) method, we analyzed various designs of optical microcavities based on a thin semiconductor membrane perforated with a hexagonal lattice of air holes. The microcavities consisted of single or multiple lattice defects formed by increasing or decreasing radii of air holes, thereby producing acceptor or donor-like defect states. The analyzed geometries include single acceptor or donor defects, as well as ring, hexagonal and triangular cavities. Properties of these structures (excluding single donor-type defect) have not been analyzed previously in a slab of finite thickness. Frequencies, quality factors and radiation patterns of localized defect modes were analyzed as a function of parameters of photonic crystal (PC) and defects. Finally, we discuss possible applications of these structures in active or passive optical devices.
\end{abstract}

Keywords: photonic crystals, finite-difference time-domain methods, optical microcavities

\section{INTRODUCTION}

By introducing point or line defects into a periodic array of holes perforating an optically thin semiconductor slab, one can construct various passive and active optical devices. This technique was employed in making a semiconductor (InGaAsP) laser $^{1}$ (emitting at $\lambda=1.55 \mu \mathrm{m}$ ), and for demonstrating Si optical waveguides with sharp bends. ${ }^{2}$ In these structures, light is confined laterally by means of distributed Bragg reflection and vertically by total internal reflection. Beside an easy fabrication procedure (compared to the fabrication of 3D PC structures), the advantage of this approach lies also in facilitating the integration of many optical components on a single chip.

Microcavity formation via alteration of the refractive index of a single defect hole in a hexagonal photonic crystal (PC) has been previously analyzed theoretically by our group. ${ }^{3}$ In that analysis, the radius of the defect was equal to that of the unperturbed PC holes, but its refractive index was tuned between one and the refractive index of the slab. It was predicted that, in the described structure, dipole defect modes can be excited with quality factors as high as 30000 . However, we recently described a more thorough analysis of this problem, ${ }^{4,5}$ where we showed that quality factors of these single defect structures were actually limited to several thousand, but that $\mathrm{Q}$ factors of more than 30000 were still achievable with proper, more sophisticated designs.

In this article we will concentrate on single defect microcavities formed by reducing or increasing the radius of a single hole in a hexagonal PC array, as well as multiple defect microcavities in shapes of triangles, hexagons or rings. Properties of these structures have not been analyzed previously in a slab of finite thickness. The thickness of the slab is $d$ and its refractive index is equal to $n_{\text {slab }}$. The horizontal plane (plane of the slab) corresponds to the $\mathrm{x}-\mathrm{y}$ plane and $z$ is the direction perpendicular to it. The interhole spacing is denoted by $a$, the radius of an individual hole by $r$ and $\lambda$ is the wavelength in air. All microcavities discussed here are based on PC with $d / a=0.65, r / a=0.3$ and $n_{\text {slab }}=3.4$. Band diagram for TE-like modes in the PC slab with these parameters is shown in Figure 1. In all our calculations, the boundary for separation of vertical from lateral loss (i.e. the vertical quality factor $Q_{\perp}$ from the lateral quality factor $Q_{\|}$) was positioned approximately at $\lambda / 2$ from the surface of the membrane. As the number of PC layers around the defect increases, $Q_{\|}$increases exponentially and the total quality factor $Q$ approaches $Q_{\perp} .{ }^{3}$ Therefore, $Q_{\perp}$ will represent the limiting value of quality factor in our structures.

Further author information: Send correspondence to Jelena Vučković. E-mail: jela@caltech.edu 


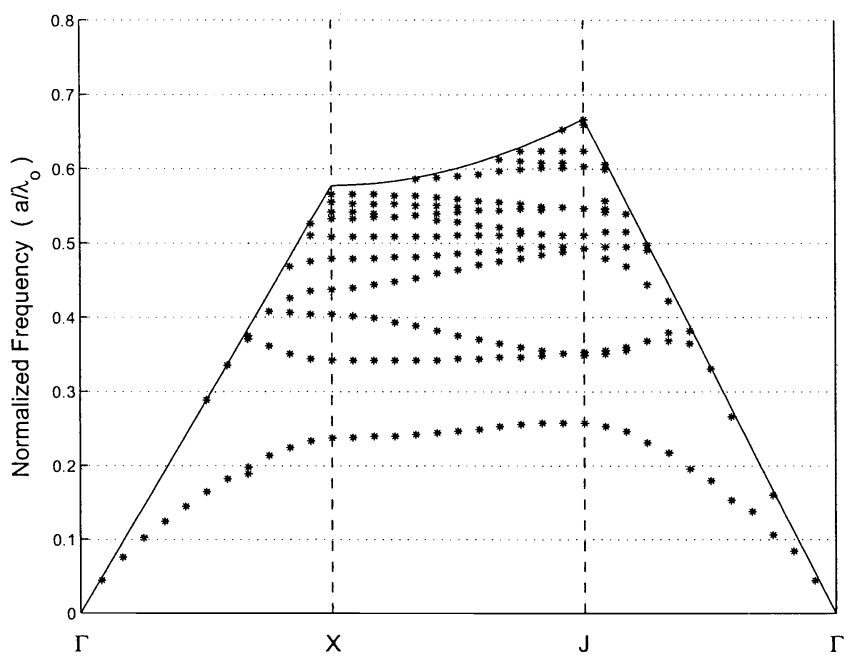

Figure 1. Band diagram for TE-like modes of a thin slab $(n=3.4, d / a=0.65)$ surrounded by air on both sides and patterned with a hexagonal array of air holes $(\mathrm{r} / \mathrm{a}=0.3)$. Band diagram is calculated using the 3D FDTD method.

\section{SINGLE DEFECT HOLE}

The simplest way of forming a microcavity is by changing the radius of a single hole. By increasing the radius of a single hole, an acceptor defect state is excited, i.e. pulled into the band-gap from the dielectric band. On the other hand, by decreasing the radius of an individual hole, a donor defect state is excited and pulled into the band gap from the air band. Acceptors tend to concentrate their electric field energy density in regions where the large refractive index was located in the unperturbed PC, while the electric field energy density of donors is concentrated in regions where there was air in the unperturbed PC, as shown in Figure 2. For laser design, donor type state is a better choice, since one could achieve a strong overlap between intense electric field and active region. Furthermore, as one could notice in Figure 2, electric field for acceptor type states is concentrated close to the edges of a large defect hole, which can lead to an increase in surface recombination. Beside that, vertical scattering at the edges of large defect holes may limit maximum quality factors in acceptor type structures. However, acceptor modes can still be very interesting for designs of passive optical components, such as filters. ${ }^{6}$

Parameters of acceptor and donor states as a function of the radius of the defect hole $\left(r_{\text {def }}\right)$ are shown in Figure 3. For all presented analyses, 5 layers of PC holes surround the defect and in the applied discretization $a=20$. Therefore, $Q$ factors of these single defect structures are limited to around 2500. We have noted this previously for donor-type single defect structures, ${ }^{4,5}$ but the same range of Qs is now obtained for acceptors. By tuning the radius of the defect hole, we also tune the frequency of the defect mode throughout the band-gap. In this process, we can optimize the quality factor of the mode. Lateral quality factors $\left(Q_{\|}\right)$can always be increased by adding more $\mathrm{PC}$ layers around the defect. On the other hand, vertical quality factors $\left(Q_{\perp}\right)$ saturate after around $5 \mathrm{PC}$ layers surrounding the defect.

\section{MICROCAVITIES CONSITING OF MULTIPLE DEFECTS}

By generating multiple defects in the $\mathrm{PC}$ structure, we can produce various shapes of microcavities. These structures will be multi-mode with increased modal volumes, but they may have advantages over single defect designs in some situations, such as for the fabrication of electrically pumped devices. However, we will see that these larger cavities actually have modest $\mathrm{Q}$ factors, limitted by vertical losses.

Let us first consider a hexagon with a side equal to $2 a$, produced by omitting multiple $\mathrm{PC}$ holes. One of the modes that could be excited in this structure is shown in Figure 4. Its parameters are $a / \lambda=0.2886, Q_{\|}=1547$ and $Q_{\perp}=713$. Mode will radiate laterally, in $6 \Gamma X$ directions. $Q_{\perp}$ is quite small, and, therefore, vertical scattering is very critical.

If we keep the central hole of this structure (in the center of hexagon), thereby producing a ring with a side equal 


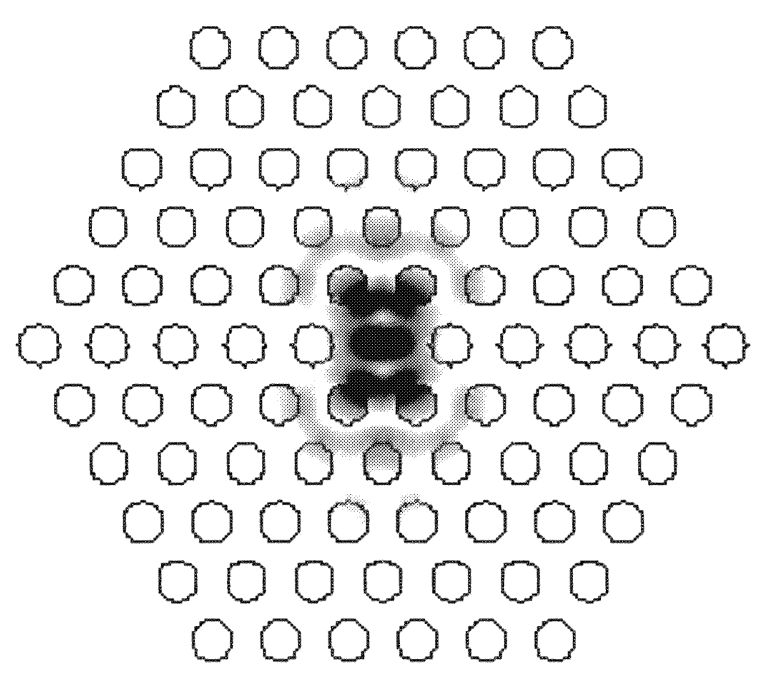

(a)

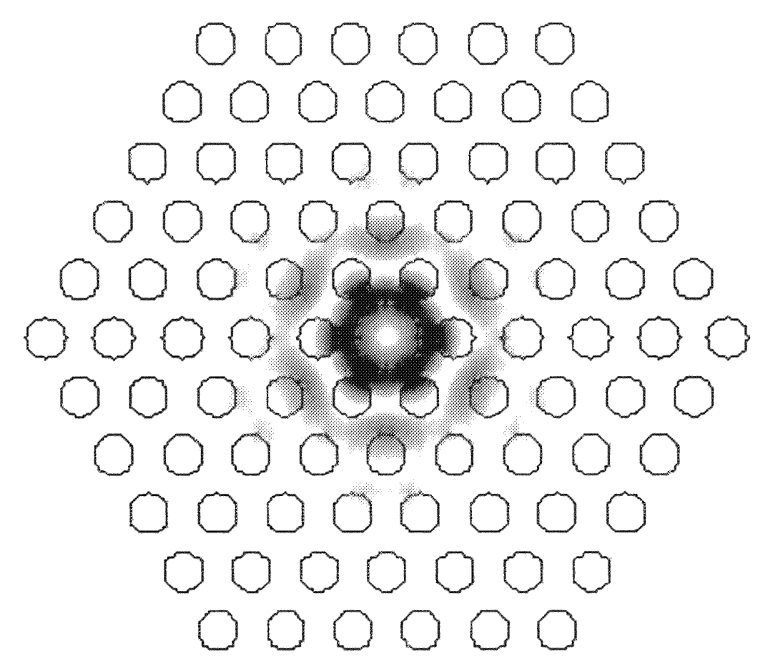

(b)

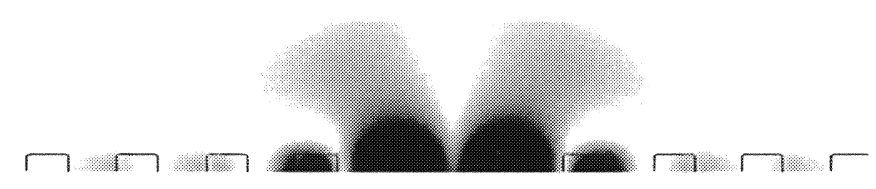

(c)

Figure 2. Electric field intensity pattern of (a) donor state (x-dipole mode); (b) and (c) acceptor defect state, excited in the hexagonal PC of air holes perforating an optically thin slab. The defect is formed by (a) omitting a single hole; (b) and (c) increasing the radius of the central hole from $r / a=0.3$ to $r / a=0.5$. The plotted intensity patterns are: (a),(b) for the $x-y$ plane in the middle of the slab; (c) for the $x-z$ half-plane in the middle of the slab.

to $2 a$, and excite the same mode as the one shown in Figure 4 , its parameters are $a / \lambda=0.29436, Q_{\|}=1581$ and $Q_{\perp}=542$. Therefore, the mode senses the central hole only weekly and the overlap with air in it slightly increases the mode's frequency. $Q_{\|}$remains unchanged, while $Q_{\perp}$ decreases a little, due to increased vertical scattering at the edges of this extra hole.

Finally, let us analyze a triangle with a side $2 a$ produced by omitting holes of the PC and the mode shown in Figure 4. Parameters of this mode are $a / \lambda=0.3086, Q_{\|}=352$ and $Q_{\perp}=365$. Therefore, both lateral and vertical losses increase in this type of the microcavity.

Certainly, a more thorough analysis of these structures should be done to carefully scan the range of PC parameters, but our first results show that these large defect structures are not really good candidates for high $\mathrm{Q}$ cavities. On the other hand, we have managed to optimize Qs of dipole defect modes to values of above 30000, by tuning parameters of $\mathrm{PC}$ and holes around the defect. ${ }^{5}$ 


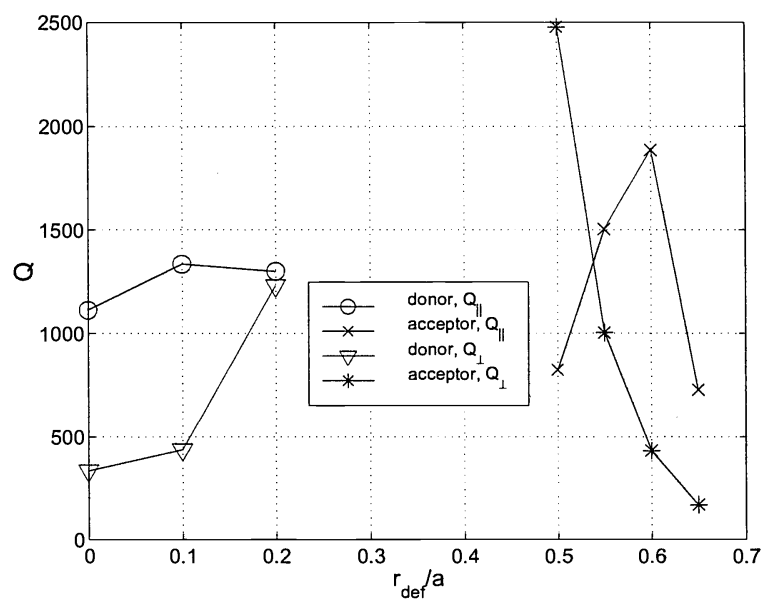

(a)

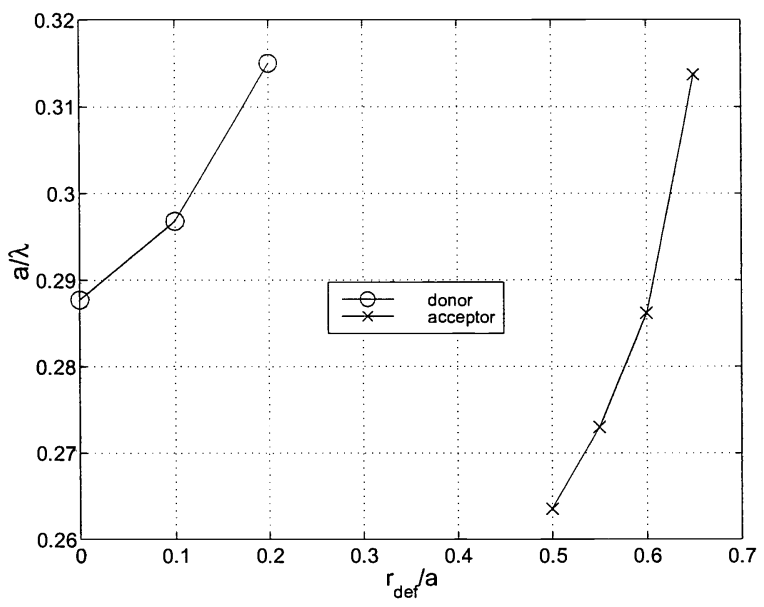

(b)

Figure 3. Parameters of the donor (dipole) and acceptor mode in the single defect structure $(r / a=0.3, d / a=0.65)$ as a function of the defect hole radius $r_{d e f}$ : (a) Q factors; (b) frequency in units $a / \lambda$;

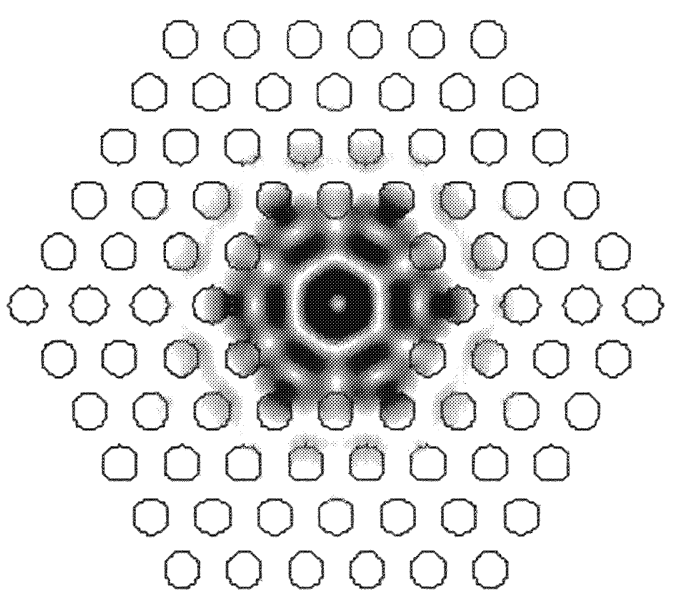

(a)

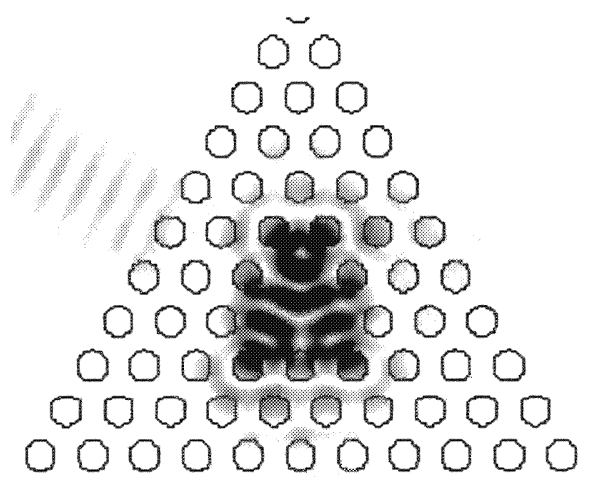

(b)

Figure 4. Electric field intensity patterns of the modes excited in the large (a) hexagonal; (b) triangular microcavity.

\section{FABRICATION}

SEM micrographs of some of discussed structures fabricated in AlGaAs are shown in Figure 5. For the detailed description of fabrication procedure of $\mathrm{PC}$ structures in $\mathrm{AlGaAs}$ or $\mathrm{Si}$, readers are referred to References 5 and 7. 


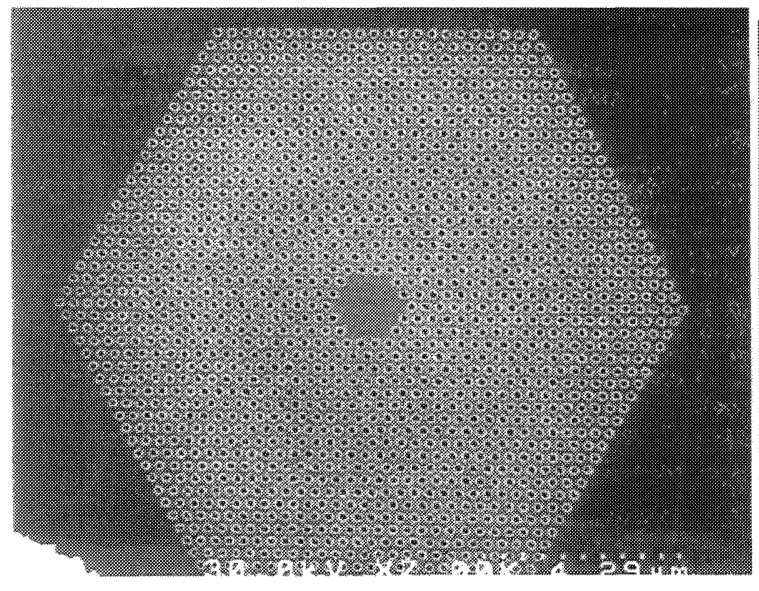

(a)

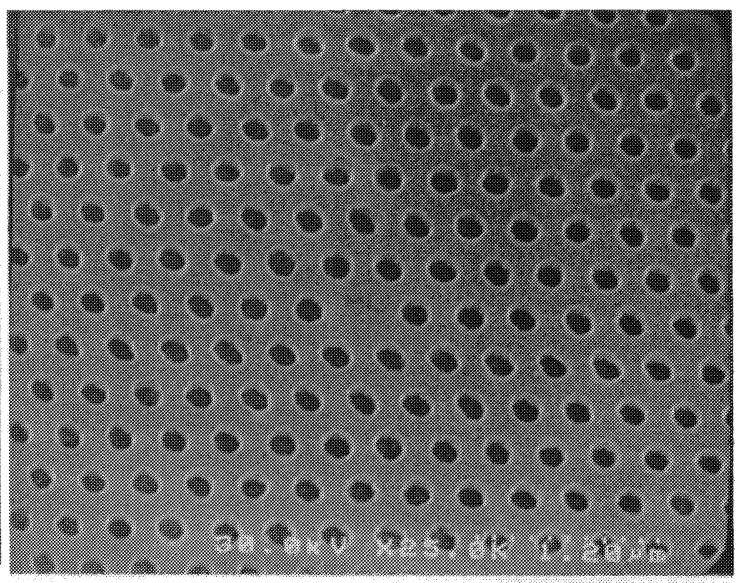

(b)

Figure 5. SEM picture showing the top views of fabricated structures.

\section{CONCLUSION}

In conclusion, we have theoretically analyzed various microcavities formed by introducing single or multiple defects into PC arrays. Analyzed structures were based on an optically thin slab perforated with a hexagonal array of air holes. The maximum calculated quality factors were around several thousand and limited by vertical losses (i.e. out of plane scattering at the edges of holes). Mode properties ( $Q$ factors, frequencies) are very sensitive to parameters of photonic crystal and defects, which can be exploited when designing various optical devices (e.g. lasers, filters). Q factor can be increased further (to values of more than 30000) with more sophisticated designs, as we have discussed in our recent work. ${ }^{4,5}$

\section{REFERENCES}

1. O. Painter, R. Lee, A. Scherer, A. Yariv, J. O'Brien, P. Dapkus, and I. Kim, "Two-Dimensional Photonic BandGap Defect Mode Laser," Science 284, pp. 1819-1821, June 1999.

2. M. Lončar, D. Nedeljković, T. Doll, J. Vučković, A. Scherer, and T. P. Pearsall, "Waveguiding at 1500nm using photonic crystal structures in silicon on insulator wafers," Applied Physics Letters 77, pp. 1937-1939, Sept. 2000.

3. O. Painter, J. Vučković, and A. Scherer, "Defect Modes of a Two-Dimensional Photonic Crystal in an Optically Thin Dielectric Slab," Journal of the Optical Society of America B 16, pp. 275-285, Feb. 1999.

4. J. Vučković, M. Lončar, H. Mabuchi, and A. Scherer, "Photonic crystal microcavities for strong coupling between an atom and the cavity field," Proceedings of the LEOS2000, Rio Grande, Puerto Rico, pp. 840-841.

5. J. Vučković, M. Lončar, H. Mabuchi, and A. Scherer, "Photonic crystal microcavities for strong coupling between an atom and the cavity field," submitted to Physical Review A .

6. S. Noda, A. Chutinan, and M. Imada, "Trapping and emission of photons by a single defect in a photonic bandgap structure," Nature 407, pp. 608-610, Oct. 2000.

7. M. Lončar, T. Doll, J. Vučković, and A. Scherer, "Design and fabrication of silicon photonic crystal optical waveguides," Journal of lightwave technology 18, pp. 1402-1411, Oct. 2000. 